\title{
Trial Inclusion and Exclusion Criteria Domain
}

National Cancer Institute

\section{Source}

National Cancer Institute. Trial Inclusion and Exclusion Criteria Domain. NCI Thesaurus.

Code C49620.

A subject domain utilized for the submission of information encompassing and

representing data, vocabulary or records related to trial inclusion and exclusion criteria. 